\title{
High-oleic rapeseed (canola) and flaxseed oils modulate serum lipids and inflammatory biomarkers in hypercholesterolaemic subjects
}

\author{
Leah G. Gillingham ${ }^{1}$, Jennifer A. Gustafson ${ }^{1}$, Song-Yee Han $^{2}$, Davinder S. Jassal ${ }^{2}$ and Peter J. H. Jones ${ }^{1 *}$ \\ ${ }^{1}$ Department of Human Nutritional Sciences, Richardson Centre for Functional Foods and Nutraceuticals, 196 Innovation \\ Drive, University of Manitoba, Winnipeg, MB, Canada R3T 6C5 \\ ${ }^{2}$ Section of Cardiology, Department of Internal Medicine, University of Manitoba, St Boniface General Hospital, Winnipeg, \\ $M B$, Canada
}

(Received 27 April 2010 - Revised 23 August 2010 - Accepted 24 August 2010 - First published online 29 September 2010)

\section{Abstract}

Recently, novel dietary oils with modified fatty acid profiles have been manufactured to improve fatty acid intakes and reduce CVD risk. Our objective was to evaluate the efficacy of novel high-oleic rapeseed (canola) oil (HOCO), alone or blended with flaxseed oil (FXCO), on circulating lipids and inflammatory biomarkers $v$. a typical Western diet (WD). Using a randomised, controlled, crossover trial, thirty-six hypercholesterolaemic subjects consumed three isoenergetic diets for $28 \mathrm{~d}$ each containing approximately $36 \%$ energy from fat, of which $70 \%$ was provided by HOCO, FXCO or WD. Dietary fat content of SFA, MUFA, PUFA $n-6$ and $n-3$ was $6,23,5,1 \%$ energy for HOCO; 6,16 , 5, 7.5\% energy for FXCO; 11.5, 16, 6, 0.5\% energy for WD. After $28 \mathrm{~d}$, compared with WD, LDL-cholesterol was reduced $15 \cdot 1 \%(P<0 \cdot 001)$ with FXCO and $7 \cdot 4 \%(P<0.001)$ with HOCO. Total cholesterol (TC) was reduced $11 \%(P<0 \cdot 001)$ with FXCO and $3.5 \%(P=0.002)$ with HOCO compared with WD. Endpoint TC differed between FXCO and HOCO $(P<0.05)$. FXCO consumption reduced HDL-cholesterol by $8.5 \%(P<0.001)$ and LDL:HDL ratio by $7.5 \%(P=0.008) v$. WD. FXCO significantly decreased E-selectin concentration compared with WD $(P=0.02)$. No differences were observed in inflammatory markers after the consumption of HOCO compared with WD. In conclusion, consumption of novel HOCO alone or when blended with flaxseed oil is cardioprotective through lipid-lowering effects. The incorporation of flaxseed oil may also target inflammation by reducing plasma E-selectin.

Key words: High-oleic rapeseed (canola) oil: Flaxseed oil: Lipids: Inflammatory biomarkers: CVD

Considerable interest has been focused on the influence of dietary fatty acids on CVD risk $^{(1)}$, with attention being centred on the value of dietary fat quality ${ }^{(2-4)}$. Evidence from prospective cohort studies and controlled clinical trials supports the use of dietary unsaturated fatty acids for the reduction of CVD risk factors ${ }^{(1-3,5)}$. Therefore, dietary guidelines with a focus on cardiovascular health have recommended replacing SFA intakes with unsaturated fats ${ }^{(6)}$. Increased consumption of novel dietary oils rich in MUFA and $\alpha$-linolenic acid (ALA) may improve the fatty acid imbalance typical of modern Western diets (WD), high in SFA and the $n-6: n-3$ fatty acid ratio ${ }^{(7)}$. Recent advances in the edible oil industry have produced dietary oils with nutritionally superior fatty acid profiles ${ }^{(8)}$. High-oleic rapeseed (canola) oil (HOCO) is rich in MUFA, low in SFA and exhibits a low ratio of $n-6: n-3$ fatty acids. With enhanced oxidative stability, HOCO is an attractive oil replacement for high SFA-high trans oil varieties currently used in the food industry. Furthermore, recommendations have been made to increase dietary $n$ - 3 fatty acid intake $^{(7)}$. Flaxseed oil is a rich source of ALA; however, as flaxseed oil is less commonly consumed, blending flaxseed oil with other dietary oils provides a viable option to increase ALA intakes in WD.

Dyslipidaemia, specifically elevated LDL-cholesterol, is a primary risk factor in predicting CVD events and a major target of dietary intervention ${ }^{(9)}$. Recently, elevated concentrations of circulating inflammatory biomarkers have been associated with cardiovascular events ${ }^{(10-12)}$. C-reactive protein (CRP) and pro-inflammatory cytokines, such as IL-6, initiate the development of atherosclerosis by up-regulating endothelial expression of adhesion molecules, including vascular cell adhesion molecule-1 (VCAM-1), intercellular adhesion molecule-1 (ICAM-1) and E-selectin ${ }^{(13)}$. Therefore,

Abbreviations: ALA, $\alpha$-linolenic acid; CRP, C-reactive protein; FXCO, flaxseed/high-oleic rapeseed (canola) oil; HOCO, high-oleic rapeseed (canola) oil; ICAM-1, intercellular adhesion molecule-1; IMT, intima-media thickness; sICAM-1, Soluble ICAM-1; sVCAM-1, soluble vascular cell adhesion molecule-1; TC, total cholesterol; WD, Western diet; VCAM-1, vascular cell adhesion molecule-1.

*Corresponding author: P. J. H. Jones, fax +1 204474 7552, email peter_jones@umanitoba.ca 
the reduction of both circulating LDL-cholesterol levels and inflammatory biomarkers is important in ameliorating CVD risk.

To date, the efficacy of HOCO consumption in modulating established biomarkers of CVD risk has not been investigated in a human clinical study. Additionally, although a high dose of flaxseed oil consumption has been reported to reduce inflammatory biomarkers in at-risk subjects ${ }^{(14)}$, the effects of flaxseed oil on serum lipids have been inconsistent ${ }^{(14,15)}$. Therefore, the objectives of this human clinical study were to evaluate the efficacy of HOCO and a flaxseed/high-oleic rapeseed oil (FXCO) blend in modulating circulating lipids and inflammatory biomarkers associated with CVD risk as compared with a typical WD.

\section{Experimental methods}

\section{Subjects}

Thirty-nine individuals (fourteen males and twenty-five females) were recruited using flyers and media advertisements. Subjects were screened for LDL-cholesterol after $12 \mathrm{~h}$ of fasting, and detailed blood chemistry analyses were performed. Inclusion criteria for the study were serum LDL-cholesterol $>3.0 \mathrm{mmol} / \mathrm{l}$, age 18-65 years and BMI between 22 and $36 \mathrm{~kg} / \mathrm{m}^{2}$. Before study enrolment, the subjects underwent a routine physical examination by the study physician. Exclusion criteria were documented atherosclerotic disease, inflammatory disease, diabetes mellitus, uncontrolled hypertension, kidney disease, cancer, tobacco smoking, use of lipidlowering medications for at least 3 months before starting the study, alcohol consumption $>2$ servings/d or excessive exercise expenditure of $>16735 \mathrm{~kJ}$ (4000 kcal)/week. The present study was conducted according to the guidelines laid down in the Declaration of Helsinki, and all procedures involving human subjects were approved by the Bannatyne Campus Research Ethics Board (Protocol no. B2007:071) and the St Boniface General Hospital Research Review Committee (Ref no. RCC/2007/0862). A written informed consent was obtained from all the subjects. The study is registered in the ClinicaTrials.gov registry (identifier \#NCT00927199).

\section{Experimental design}

A randomised, single-blind, crossover, controlled diet clinical trial was conducted at the Clinical Research Unit at the Richardson Centre for Functional Foods and Nutraceuticals, University of Manitoba. The study was designed as three phases with $28 \mathrm{~d}$ per phase separated by $4-8$-week washout periods during which the subjects consumed their habitual diets. The subjects were randomised to the three experimental diets using a $3 \times 3$ Latin-square design. Diets were individualised to meet daily energy requirements for weight maintenance for each subject as determined by the Mifflin equation ${ }^{(16)}$, multiplied by a factor of 1.7 for medium physical activity. The study diets were prepared in the metabolic kitchen of the Richardson Centre Clinical Nutrition Research Unit and the food ingredients were weighed within $0.5 \mathrm{~g}$. Diets consisted of three isoenergetic meals prepared according to a $3 \mathrm{~d}$ cycle menu providing a variety of foods. In order to ensure stability of the flaxseed oil, experimental oils were added to cold foods; provided in milkshakes at breakfast and puddings at lunch and dinner. The subjects consumed one of three daily meals (breakfast) at the Richardson Centre Clinical Nutrition Research Unit under supervision, while other meals (lunch and dinner) were prepared and cold packed for take out. The subjects were instructed to consume only foods and beverages provided by the Richardson Centre Clinical Nutrition Research Unit and to refrain from alcoholic and caffeinated beverages during the intervention periods. The subjects were advised to maintain their typical physical activity level and asked to report any symptoms or changes in health and medications throughout the study. Subjects' body weights were measured under supervision every morning before breakfast using a medical scale (Detecto, Webb City, MO, USA) to monitor weight stability.

\section{Diets}

Experimental diets were designed as typical WD containing $50 \%$ of energy as carbohydrate, $15 \%$ as protein and $35 \%$ as fat, of which $70 \%$ was provided by the experimental oil. Diets were identical in composition throughout each phase, except for the type of experimental oil. Macronutrient profiles of experimental diets (Table 1) were analysed using the nutrient composition software FOOD PROCESSOR (Food Processor version 7.81; Salem, OR, USA). Experimental oils tested included (1) HOCO (approximately 70\% oleic acid; Canola Harvest HiLo ${ }^{\circledR}$; Richardson Oilseed Limited, Lethbridge, AB, Canada); (2) a 1:1 blend of the HOCO and flaxseed oil (FXCO) (approximately 55\% ALA and no lignans; Bioriginal Food \& Science Corporation, Saskatoon, SK, Canada); (3) a blend of oils typical of a WD including non-salted butter (12\%), extra-virgin olive oil (35\%), vegetable lard (35\%) and sunflower oil (>60\% linoleic acid) (18\%). Fatty acid profiles of experimental oils are reported in Table 2 .

\section{Blood sampling and serum lipid analysis}

On days 1, 2, 28 and 29 of each phase, $12 \mathrm{~h}$ fasted serum and EDTA plasma samples were collected. Within $1 \mathrm{~h}$ of blood collection, serum, plasma and erythrocyte fractions were separated by centrifugation at $3000 \mathrm{rpm}$ for $20 \mathrm{~min}$ at $4{ }^{\circ} \mathrm{C}$, aliquoted and immediately stored at $-80^{\circ} \mathrm{C}$ until further analysis.

Serum total cholesterol (TC), HDL-cholesterol, TAG and glucose levels were determined by automated enzymatic 
Table 1. Macronutrient profile of the three experimental diets*

\begin{tabular}{|c|c|c|c|c|c|c|}
\hline & \multicolumn{2}{|c|}{ Western diet } & \multicolumn{2}{|c|}{$\begin{array}{l}\text { High-oleic rapeseed } \\
\text { oil diet }\end{array}$} & \multicolumn{2}{|c|}{$\begin{array}{l}\text { Flaxseed and high-oleic } \\
\text { rapeseed oil diet }\end{array}$} \\
\hline & $g / d$ & $\%$ & $g / d$ & $\%$ & $g / d$ & $\%$ \\
\hline Energy (kJ/d) & \multicolumn{2}{|c|}{10460} & \multicolumn{2}{|c|}{10460} & \multicolumn{2}{|c|}{10460} \\
\hline Carbohydrate & 305 & $48 \cdot 8$ & 305 & $48 \cdot 8$ & 304 & $48 \cdot 7$ \\
\hline Fibre & 20 & $3 \cdot 3$ & 20 & $3 \cdot 2$ & 20 & $3 \cdot 2$ \\
\hline Protein & 90 & $14 \cdot 4$ & 90 & 14.4 & 90 & 14.4 \\
\hline Fat & 102 & $36 \cdot 8$ & 102 & $36 \cdot 8$ & 103 & 36.9 \\
\hline SFA & $31 \cdot 2$ & $11 \cdot 2$ & $15 \cdot 7$ & $5 \cdot 6$ & $17 \cdot 0$ & $6 \cdot 1$ \\
\hline MUFA & $44 \cdot 8$ & $16 \cdot 1$ & 63.5 & 22.9 & $44 \cdot 2$ & $15 \cdot 9$ \\
\hline PUFA & $18 \cdot 0$ & 6.5 & 15.9 & 5.7 & $34 \cdot 1$ & $12 \cdot 3$ \\
\hline $18: 2 n-6$ & $16 \cdot 5$ & $5 \cdot 9$ & $13 \cdot 3$ & $4 \cdot 8$ & $13 \cdot 5$ & 4.9 \\
\hline $18: 3 n-3$ & $1 \cdot 3$ & 0.5 & $2 \cdot 4$ & 0.8 & 20.6 & $7 \cdot 4$ \\
\hline$n-6: n-3$ ratio & \multirow{2}{*}{\multicolumn{2}{|c|}{$\begin{array}{c}12 \cdot 8 \\
201.1\end{array}$}} & \multirow{2}{*}{\multicolumn{2}{|c|}{$\begin{array}{c}5 \cdot 5 \\
169 \cdot 8\end{array}$}} & \multirow{2}{*}{\multicolumn{2}{|c|}{$\begin{array}{c}0.7 \\
169.4\end{array}$}} \\
\hline Cholesterol (mg/d) & & & & & & \\
\hline
\end{tabular}

* The macronutrient profile of the three experimental diets was estimated using FOOD PROCESSOR software (version 7.81; Food Processor, Salem, OR, USA).

methods on a Vitros-350 chemistry analyser (Ortho-Clinical Diagnostics, Markham, ON, Canada). Serum LDL-cholesterol levels were calculated by the Friedewald equation ${ }^{(17)}$.

\section{Plasma inflammatory biomarker and cell adhesion} molecule analysis

Plasma CRP levels were measured using quantitative colorimetric sandwich ELISA according to the manufacturer's guidelines (R\&D Systems, Minneapolis, MN, USA). IL-6 levels were measured by high-sensitivity ELISA (R\&D Systems, Minneapolis, MN, USA). The intra-assay and interassay CV values were 2.31 and $4.26 \%$, and 2.51 and $8.04 \%$, for CRP and IL-6, respectively.

Plasma soluble adhesion molecules (sVCAM-1, sICAM-1 and sE-selectin) were measured simultaneously by flow cytometry using multianalyte profiling performed on a Luminex-100 IS system (Luminex Corporation, Austin, TX, USA). Plasma concentrations of sVCAM-1, SICAM-1 and sE-selectin were determined using a MILLIPLEX MAP human CVD panel-1 (3-plex) kit according to the manufacturer's guidelines (HCVD1-67AK; Millipore Corporation,
Billerica, MA, USA). Acquired median fluorescent intensity data were analysed using a weighted 5-parameter logistic curve by the IS 2.3 software (Luminex Corporation, Austin, TX, USA). The sensitivity of the assay reported by the manufacturer had a minimum detectable concentration of 0.016, 0.009 and $0.079 \mathrm{ng} / \mathrm{ml}$ for sVCAM-1, sICAM-1 and sE-selectin, respectively. The intra-assay and inter-assay $\mathrm{CV}$ values were 7.4 and $10.9 \%, 8.8$ and $11.3 \%$, and 6.0 and $7 \cdot 4 \%$ for sVCAM-1, sICAM-1 and sE-selectin, respectively.

For the analyses of inflammatory biomarkers by ELISA and adhesion molecules by LUMINEX, controls (low, medium and high) supplied by the respective assay manufacturer and the subject plasma samples were assayed in duplicate by a single laboratory technician with all the samples for each subject run in one assay.

\section{Plasma fatty acid profile}

Plasma total lipids were extracted by the Folch method $^{(18)}$ using chloroform-methanol (2:1, v/v) containing $0.01 \%$ BHT (Sigma-Aldrich, Oakville, ON, Canada) and

Table 2. Fatty acid composition of the three experimental dietary oils*

\begin{tabular}{|c|c|c|c|}
\hline Fatty acid & $\begin{array}{c}\text { Western diet } \\
\text { ( } \mathrm{g} / 100 \mathrm{~g} \text { total fatty acids) }\end{array}$ & $\begin{array}{l}\text { High-oleic rapeseed oil diet } \\
\text { (g/100 g total fatty acids) }\end{array}$ & $\begin{array}{l}\text { Flaxseed and high-oleic rapeseed } \\
\text { oil diet (g/100 g total fatty acids) }\end{array}$ \\
\hline$\Sigma S F A$ & $28 \cdot 6$ & $6 \cdot 6$ & 7.5 \\
\hline $10: 0$ & 0.3 & - & - \\
\hline $12: 0$ & 0.4 & - & - \\
\hline $14: 0$ & 1.7 & - & - \\
\hline $16: 0$ & $18 \cdot 2$ & 3.9 & 4.6 \\
\hline $18: 0$ & $7 \cdot 6$ & $1 \cdot 8$ & $2 \cdot 7$ \\
\hline $20: 0$ & 0.3 & 0.6 & 0.3 \\
\hline$\Sigma M U F A$ & $48 \cdot 3$ & $75 \cdot 2$ & $44 \cdot 1$ \\
\hline $16: 1 n-7$ & $1 \cdot 4$ & 0.2 & - \\
\hline $18: 1 n-9$ & $46 \cdot 5$ & $73 \cdot 7$ & 43.4 \\
\hline $20: 1 n-9$ & 0.4 & $1 \cdot 3$ & 0.7 \\
\hline$\Sigma P U F A$ & $22 \cdot 6$ & $17 \cdot 9$ & $48 \cdot 4$ \\
\hline $18: 2 n-6$ & $21 \cdot 7$ & $16 \cdot 3$ & $15 \cdot 9$ \\
\hline $18: 3 n-3$ & 0.8 & 1.7 & 32.4 \\
\hline
\end{tabular}

-, Undetected fatty acid.

* Values were determined by GC of triplicate samples of the dietary oil blends. 
heptadecanoic acid as an internal standard (Sigma-Aldrich, Oakville, ON, Canada). Extracted fatty acids were methylated with methanolic $\mathrm{HCl}$. Fatty acid methyl esters were separated on a Supelcowax 10 column $(30 \mathrm{~m} \times 0.25 \mathrm{~mm}$ with $0.25 \mu \mathrm{m}$ film thickness; Supelco, Bellefonte, PA, USA) using an Agilent $6890 \mathrm{~N}$ gas chromatograph equipped with a flame ionisation detector (Agilent Technologies, Mississauga, ON, Canada). The oven was programmed from 70 to $240^{\circ} \mathrm{C}$ in four temperature steps $\left(70^{\circ} \mathrm{C}\right.$ for $1 \mathrm{~min}$, rise of $25^{\circ} \mathrm{C} / \mathrm{min}, 180^{\circ} \mathrm{C}$ for $2 \mathrm{~min}$, rise of $3^{\circ} \mathrm{C} / \mathrm{min}, 220^{\circ} \mathrm{C}$ for $10 \mathrm{~min}$, rise of $20^{\circ} \mathrm{C} / \mathrm{min}, 240^{\circ} \mathrm{C}$ for $15 \mathrm{~min}$ ). Samples were run with a 10:1 split ratio, and He was used as the carrier gas with a column flow rate of $1.0 \mathrm{ml} / \mathrm{min}$. Temperatures for the injector and detector were set at 280 and $300^{\circ} \mathrm{C}$, respectively. Individual fatty acids were identified by comparison with known standards (NuChek Prep, Inc., Elysian, MN, USA). Individual fatty acids were calculated according to the peak area relative to the total area and expressed as the percentage of total fatty acids.

\section{Intima-media thickness assessment}

A subset of study subjects ( $n$ 18; randomised selection from study population) underwent clinical endothelial health assessment at the onset of the study (phase 1; days 1-3) and at the end of each treatment phase (days 24-26) by common carotid arterial ultrasound to assess changes in intima-media thickness (IMT). Assessment of common carotid IMT was performed with the use of an annular array ultrasound imaging system (9L probe; GE Vivid 7, Milwaukee, IL, USA). The subjects were examined in the supine position. Ultrasound scans of the right and left common carotid arteries were performed at the bifurcation of the first proximal centre of internal carotid arteries as described previously ${ }^{(19)}$. All the measurements were made offline of the longitudinal carotid IMT scans using dedicated computer software (GE Echopac BT 08; Milwaukee, IL, USA). Average and maximal IMT values of each segment were measured as described previously ${ }^{(19)}$. All ultrasound scans were performed by two trained sonographers, and the recorded ultrasound images were analysed blindly at the Institute of Cardiovascular Sciences, St Boniface General Hospital Research Centre, Winnipeg, Canada.

\section{Statistical analyses}

Statistical analysis was performed using SPSS 16.0 (SPSS, Inc., Chicago, IL, USA). Results are expressed as means with their standard errors unless otherwise noted. For variables with non-normal distribution, as determined by a Shapiro-Wilk test value $<0.05$, statistical analyses were conducted after a logarithmic (base 10) transformation. Data on inflammatory biomarkers and adhesion molecules were not normally distributed and are reported as the median and 25th and 75th percentiles. Effects of dietary treatment were examined using a mixed model ANOVA procedure with diet, sequence and phase as fixed factors and subject as a random factor in the model. Baseline values were inserted into the model as covariates for serum lipid measurements. Significant diet effects were examined with the Bonferroni adjustment for multiple comparisons. For serum lipids, percentage change from baseline for each group was analysed with a two-tailed paired Student's $t$ test. Pearson correlation analyses were conducted to test the associations between lipid levels and inflammatory biomarkers. Statistical significance was set at $P<0.05$ for all the analyses. For all the data, baseline and endpoint values are reported as averages of days 1 and 2 , and days 28 and 29, respectively.

\section{Results}

\section{Subject characteristics}

Baseline characteristics of the subjects who completed the study are presented in Table 3. Thirty-six subjects (thirteen males and twenty-three females; five premenopausal) completed the study. Two subjects withdrew from the study due to relocation of residence, and one withdrew due to work-related issues. All the subjects showed good tolerance to experimental diets and reported consuming all meals provided to them. No side effects were associated with the experimental diets. The subjects reported no change in physical activity, and no significant differences were observed in body weight after the consumption of the three experimental diets.

Table 3. Baseline characteristics of the subjects

(Mean values and standard deviations; median values with their 25th and 75th percentiles)

\begin{tabular}{|c|c|c|}
\hline & Mean & SD \\
\hline \multicolumn{3}{|c|}{ Anthropometric and serum lipid measurements ( $n$ 36) } \\
\hline Age (years) & 47.49 & 11.93 \\
\hline Body wt (kg) & $78 \cdot 80$ & $17 \cdot 09$ \\
\hline Height $(\mathrm{cm})$ & $165 \cdot 50$ & $9 \cdot 78$ \\
\hline BMI $\left(\mathrm{kg} / \mathrm{m}^{2}\right)$ & 28.56 & $4 \cdot 62$ \\
\hline Total cholesterol (mmol/l) & $5 \cdot 94$ & 1.03 \\
\hline LDL-cholesterol (mmol/l' & $3 \cdot 70$ & 0.95 \\
\hline HDL-cholesterol (mmol/l) & 1.41 & 0.35 \\
\hline \multirow[t]{2}{*}{ TAG $(\mathrm{mmol} / \mathrm{l})$} & $1 \cdot 84$ & 1.09 \\
\hline & Median & 25th-75th percentile \\
\hline \multicolumn{3}{|c|}{ Plasma inflammatory biomarkers ( $n 36)$} \\
\hline C-reactive protein (mg/l) & 1.34 & $0.66-2.65$ \\
\hline IL-6 (pg/ml) & 1.59 & $1.02-2.22$ \\
\hline sVCAM-1 (ng/ml) & 1073.46 & $915 \cdot 28-1215 \cdot 78$ \\
\hline sICAM-1 (ng/ml) & 148.09 & $134 \cdot 96-159 \cdot 50$ \\
\hline \multirow[t]{2}{*}{ sE-selectin (ng/ml) } & $28 \cdot 74$ & $19 \cdot 55-36 \cdot 25$ \\
\hline & Mean & SD \\
\hline \multicolumn{3}{|c|}{ Carotid intima-media thickness (n 16) } \\
\hline Average $(\mathrm{mm})$ & 0.61 & 0.10 \\
\hline Maximum (mm) & 0.70 & 0.11 \\
\hline
\end{tabular}

sVCAM-1, soluble vascular cell adhesion molecule-1; sICAM-1, soluble intercellular adhesion molecule-1; sE-selectin, soluble E-selectin. 


\section{Plasma fatty acids}

After consumption of the experimental diets, changes in the plasma fatty acid concentrations (Table 4) reflected the fatty acid profile of the experimental diets (Table 1), verifying subjects' compliance with the experimental diets. As expected, plasma total MUFA, specifically 18:1n-9, was higher after the consumption of the HOCO diet than after the consumption of both the FXCO diet $(P<0.001)$ and the WD control $(P<0.001)$. Plasma total PUFA and total $n-3$ PUFA (including $18: 3 n-3$, $20: 5 n-3$ and $22: 5 n-3)$ were higher after the consumption of the FXCO diet than after the consumption of both the HOCO diet $(P<0.001)$ and the WD control $(P<0 \cdot 001)$. No change in plasma DHA $(22: 6 n-3)$ content was observed after the consumption of the FXCO diet compared with the WD control $(P=0.683)$; however, there was a slight decrease in plasma DHA content after the consumption of the FXCO diet compared with the HOCO diet $(P=0 \cdot 025)$. Plasma total SFA, total $n-6$ PUFA (specifically $18: 2 n-6$ ) and $n-6: n-3$ ratios were lower after the consumption of both the HOCO and FXCO diets than after the consumption of the WD control $(P<0.001$ for all). Furthermore, plasma $n-6: n-3$ ratio was lower after the consumption of the FXCO diet than after the consumption of the HOCO diet $(P<0 \cdot 001)$. No significant differences in the baseline fatty acid concentrations across the groups indicated no carryover effect and adequate washout periods between the treatment phases (data not shown).

\section{Serum lipid concentrations}

The concentrations of fasting serum lipids and glucose at the end of each treatment phase are presented in Table 5 . Serum lipid percentage change from baseline is presented in Fig. 1. After the $28 \mathrm{~d}$ treatment phase, serum TC concentrations were reduced when the subjects consumed the HOCO diet $(5.27(\operatorname{sem} 0.14) \mathrm{mmol} / \mathrm{l} ; P<0.001)$ and the FXCO diet $(5 \cdot 12($ sem $0 \cdot 13) \mathrm{mmol} / \mathrm{l} ; P<0.001)$ compared with the WD control $(5.65$ (SEM $0 \cdot 16) \mathrm{mmol} / \mathrm{l})$. TC percentage change from baseline was reduced by $3.5 \%$ $(P=0.002)$ and $11.0 \%(P<0.001)$ when subjects consumed the HOCO and FXCO diets, respectively, compared with the WD control. Furthermore, TC endpoint values $(P=0.025)$ and percentage change from baseline $(7.5 \%$; $P=0.015)$ were lower when the subjects consumed the FXCO diet compared with the HOCO diet.

Similarly, endpoint serum LDL-cholesterol concentrations were reduced after the consumption of the HOCO diet $(3 \cdot 10($ SEm $0 \cdot 12) \mathrm{mmol} / \mathrm{l} ; P<0 \cdot 001)$ and the FXCO diet $(3.08($ SEM 0.12$) \mathrm{mmol} / \mathrm{l} ; P<0.001)$ compared with the WD control (3.53 (SEM 0.14) mmol/l). LDLcholesterol percentage change from baseline was reduced by $7 \cdot 4 \% \quad(P<0.001)$ and $15.1 \%(P<0.001)$ after the consumption of the HOCO and FXCO diets, respectively, compared with the WD control. However, no differences were observed in endpoint or percentage change from baseline in LDL-cholesterol concentrations between the FXCO and HOCO diets.

Table 4. Plasma fatty acid concentration at the end of each of the three experimental diets (Mean values with their standard errors)

\begin{tabular}{|c|c|c|c|c|c|c|c|}
\hline \multirow[b]{2}{*}{ Total fatty acid (\%) (n 36) } & \multicolumn{2}{|c|}{ Western diet } & \multicolumn{2}{|c|}{$\begin{array}{l}\text { High-oleic rapeseed } \\
\text { oil diet }\end{array}$} & \multicolumn{2}{|c|}{$\begin{array}{c}\text { Flaxseed and } \\
\text { high-oleic rapeseed } \\
\text { oil diet }\end{array}$} & \multirow[b]{2}{*}{$P^{*}$} \\
\hline & Mean & SEM & Mean & SEM & Mean & SEM & \\
\hline$\Sigma S F A$ & $28 \cdot 46^{a}$ & 0.29 & $26 \cdot 30^{\mathrm{b}}$ & 0.31 & $26 \cdot 80^{\mathrm{b}}$ & 0.34 & $<0.001$ \\
\hline $14: 0$ & $0.72^{\mathrm{a}}$ & 0.04 & $0.73^{\mathrm{a}}$ & 0.04 & $0.69^{a}$ & 0.04 & 0.473 \\
\hline $16: 0$ & $19 \cdot 77^{\mathrm{a}}$ & 0.25 & $18 \cdot 33^{\mathrm{b}}$ & 0.27 & $18 \cdot 37^{\mathrm{b}}$ & 0.29 & $<0.001$ \\
\hline $18: 0$ & $6 \cdot 94^{\mathrm{a}}$ & 0.13 & $6 \cdot 27^{b}$ & 0.12 & $6 \cdot 82^{\mathrm{a}}$ & 0.14 & $<0.001$ \\
\hline ¿MUFA & $26 \cdot 18^{\mathrm{a}}$ & 0.49 & $30 \cdot 93^{b}$ & 0.58 & $26 \cdot 13^{\mathrm{a}}$ & 0.49 & $<0.001$ \\
\hline $16: 1 n-7$ & $1.65^{\mathrm{a}}$ & 0.08 & $1.53^{a, b}$ & 0.08 & $1.51^{\mathrm{b}}$ & 0.08 & 0.028 \\
\hline $18: 1 n-9$ & $21 \cdot 94^{\mathrm{a}}$ & 0.44 & $26 \cdot 45^{\mathrm{b}}$ & 0.58 & $21 \cdot 87^{\mathrm{a}}$ & 0.45 & $<0.001$ \\
\hline $18: 1 n-7$ & $1.68^{\mathrm{a}}$ & 0.04 & $1.90^{\mathrm{b}}$ & 0.07 & $1 \cdot 75^{\mathrm{a}}$ & 0.04 & $<0.001$ \\
\hline ¿PUFA & $43 \cdot 01^{a}$ & 0.61 & $40 \cdot 38^{b}$ & 0.62 & $44.55^{c}$ & 0.58 & $<0.001$ \\
\hline$\sum n-6$ PUFA & $39.68^{a}$ & 0.60 & $36 \cdot 85^{\mathrm{b}}$ & 0.60 & $36 \cdot 13^{\mathrm{b}}$ & 0.58 & $<0.001$ \\
\hline $18: 2 n-6$ & $29 \cdot 95^{\mathrm{a}}$ & 0.51 & $27 \cdot 33^{b}$ & 0.49 & $28.73^{c}$ & 0.50 & $<0.001$ \\
\hline $18: 3 n-6$ & $0.44^{\mathrm{a}}$ & 0.03 & $0.48^{\mathrm{a}}$ & 0.03 & $0.29^{b}$ & 0.02 & $<0.001$ \\
\hline $20: 3 n-6$ & $1.67^{\mathrm{a}}$ & 0.06 & $1.67^{\mathrm{a}}$ & 0.05 & $1.08^{b}$ & 0.04 & $<0.001$ \\
\hline $20: 4 n-6$ & $6 \cdot 90^{\mathrm{a}}$ & 0.24 & $6 \cdot 70^{\mathrm{a}}$ & 0.23 & $5 \cdot 49^{\mathrm{b}}$ & 0.18 & $<0.001$ \\
\hline$\Sigma n-3$ PUFA & $3 \cdot 32^{\mathrm{a}}$ & 0.07 & $3.54^{\mathrm{a}}$ & 0.08 & $8 \cdot 42^{b}$ & 0.21 & $<0.001$ \\
\hline $18: 3 n-3$ & $0.74^{a}$ & 0.03 & $0.84^{a}$ & 0.03 & $4.46^{\mathrm{b}}$ & 0.18 & $<0.001$ \\
\hline $20: 5 n-3$ & $0.54^{\mathrm{a}}$ & 0.03 & $0.62^{\mathrm{a}}$ & 0.04 & $1 \cdot 74^{b}$ & 0.11 & $<0.001$ \\
\hline $22: 5 n-3$ & $0.54^{\mathrm{a}}$ & 0.02 & $0.54^{a}$ & 0.02 & $0.75^{b}$ & 0.03 & $<0.001$ \\
\hline $22: 6 n-3$ & $1.50^{\mathrm{a}, \mathrm{b}}$ & 0.05 & $1.54^{\mathrm{a}}$ & 0.05 & $1.47^{\mathrm{b}}$ & 0.04 & 0.030 \\
\hline$n-6: n-3$ & $12 \cdot 10^{\mathrm{a}}$ & 0.28 & $10.59^{b}$ & 0.28 & $4.43^{c}$ & 0.17 & $<0.001$ \\
\hline
\end{tabular}

${ }^{\mathrm{a}, \mathrm{b}, \mathrm{c}}$ Mean values within a row with unlike superscript letters were significantly different between treatment groups $(P<0.05)$.

* $P$ values are shown for the treatment effect analysed by mixed model ANOVA (with the Bonferroni adjustment for multiple comparisons). 
Table 5. Serum lipid and glucose concentrations at the end of each of the three experimental diets (Mean values with their standard errors)

\begin{tabular}{|c|c|c|c|c|c|c|c|}
\hline \multirow[b]{2}{*}{ Serum lipids ( $n$ 36) } & \multicolumn{2}{|c|}{ Western diet } & \multicolumn{2}{|c|}{$\begin{array}{l}\text { High-oleic rapeseed } \\
\text { oil diet }\end{array}$} & \multicolumn{2}{|c|}{$\begin{array}{c}\text { Flaxseed and } \\
\text { high-oleic rapeseed } \\
\text { oil diet }\end{array}$} & \multirow[b]{2}{*}{$P^{*}$} \\
\hline & Mean & SEM & Mean & SEM & Mean & SEM & \\
\hline Total cholesterol (mmol/l) & $5 \cdot 65^{\mathrm{a}}$ & 0.16 & $5 \cdot 27^{b}$ & 0.14 & $5 \cdot 12^{\mathrm{c}}$ & 0.13 & $<0.001$ \\
\hline LDL-cholesterol (mmol/l) & $3.53^{\mathrm{a}}$ & 0.14 & $3 \cdot 10^{\mathrm{b}}$ & 0.12 & $3.08^{b}$ & 0.12 & $<0.001$ \\
\hline HDL-cholesterol ( $\mathrm{mmol} / \mathrm{l})$ & $1.37^{\mathrm{a}}$ & 0.06 & $1.33^{\mathrm{a}}$ & 0.06 & $1 \cdot 28^{\mathrm{b}}$ & 0.06 & $<0.001$ \\
\hline TAG $(\mathrm{mmol} / \mathrm{l})$ & 1.63 & $0 \cdot 16$ & $1 \cdot 84$ & $0 \cdot 18$ & 1.65 & 0.14 & 0.060 \\
\hline Total:HDL-cholesterol & 4.37 & 0.23 & $4 \cdot 24$ & 0.22 & $4 \cdot 32$ & 0.24 & 0.267 \\
\hline LDL:HDL-cholesterol & $2 \cdot 76^{\mathrm{a}}$ & 0.17 & $2 \cdot 49^{\mathrm{b}}$ & 0.14 & $2 \cdot 62^{\mathrm{b}}$ & 0.17 & $<0.001$ \\
\hline Non-HDL-cholesterol (mmol/l) & $4 \cdot 28^{a}$ & 0.17 & $3.94^{b}$ & 0.14 & $3 \cdot 84^{\mathrm{c}}$ & 0.14 & $<0.001$ \\
\hline Glucose $(\mathrm{mmol} / \mathrm{l})$ & 5.04 & $0 \cdot 16$ & 4.99 & 0.15 & 4.97 & 0.13 & 0.328 \\
\hline
\end{tabular}

a,b,c Mean values within a row with unlike superscript letters were significantly different between treatment groups $(P<0.05)$.

* $P$ values are shown for the treatment effect analysed by mixed model ANOVA (with the Bonferroni adjustment for multiple comparisons).

No differences were observed in endpoint TAG concentrations between the treatment groups $(P=0.060$; trend). With respect to percentage change from baseline, no differences were observed for serum TAG concentrations between the treatment groups.

Endpoint serum HDL-cholesterol concentrations were reduced after the consumption of the FXCO diet (1.28 (SEM 0.06) $\mathrm{mmol} / \mathrm{l}$ ) compared with the HOCO diet (1.33 (sem 0.06$) \mathrm{mmol} / \mathrm{l} ; P=0.008)$ and the WD control $(1.37$ (SEM 0.06$) \mathrm{mmol} / 1 ; P<0.001)$. The FXCO diet reduced HDL-cholesterol concentrations from baseline by $6.6 \%$ $(P=0.006)$ and $8.5 \% \quad(P<0.001)$ compared with the HOCO diet and WD control, respectively. No differences were observed in endpoint or percentage change from baseline in HDL-cholesterol concentrations between the HOCO diet and WD control.

Endpoint LDL:HDL-cholesterol ratios were reduced after the consumption of the HOCO diet (2.49 (SEM 0.14);
$P<0 \cdot 001)$ and FXCO diet (2.62 (sem 0.17); $P=0.018)$ compared with the WD control (2.76 (SEm 0.17)). Both the HOCO and FXCO diets reduced the LDL:HDL-cholesterol ratio from baseline by $5.7 \% \quad(P=0.002)$ and $7.5 \%$ $(P=0.008)$, respectively, compared with the WD control. Endpoint and percentage change from baseline in serum TC:HDL-cholesterol ratios did not differ after the treatment periods. Endpoint non-HDL-cholesterol was reduced after the consumption of the HOCO diet (3.94 (SEM 0.14); $P=0.003)$ and FXCO diet (3.84 (sem 0.14$) ; P<0.001)$ compared with the WD control (4.28 (SEM 0.17)). Both the HOCO and FXCO diets reduced non-HDL-cholesterol from baseline by $3.9 \%(P=0.004)$ and $11.7 \%(P<0.001)$, respectively, compared with the WD control. Furthermore, non-HDL-cholesterol endpoint values $(P=0.031)$ and percentage change from baseline $(7.8 \% ; P=0.030)$ were lower when the subjects consumed the FXCO diet compared with the HOCO diet.

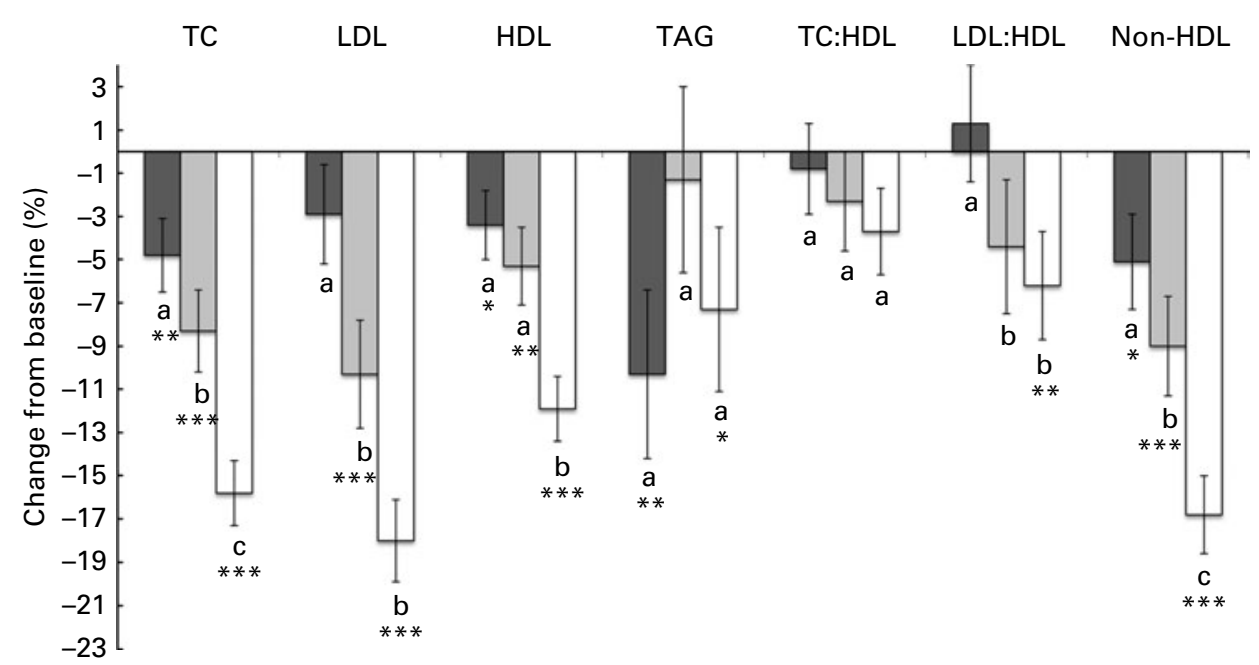

Fig. 1. Percentage change in serum lipids from baseline in response to the three treatment diets: $\mathbf{\square}$, Western diet; $\square$, high-oleic rapeseed oil diet; $\square$, flaxseed/ high-oleic rapeseed oil diet. Values are presented as means with their standard errors $(n 36)$. ${ }^{\text {a,b,c }}$ Mean values with unlike letters between treatment groups are significantly different at $P \leq 0.05$ (mixed model ANOVA followed by the Bonferroni adjustment for multiple comparisons). Mean values were significantly different when compared within treatment group from baseline: ${ }^{\star} P \leq 0.05,{ }^{\star \star} P \leq 0.01$, ${ }^{\star \star \star} P \leq 0.001$ (two-tailed paired-Student's $t$ test). TC, total cholesterol. 
No significant effects were observed in fasting serum glucose endpoint levels between the treatment groups, nor were changes from baseline values observed.

\section{Plasma inflammatory biomarkers and adhesion molecule concentrations}

The results for measures of inflammatory biomarkers by ELISA and adhesion molecules by LUMINEX were within the detection limits of the assay. No significant differences were observed in endpoint concentrations for CRP or IL-6 between the treatment groups (Table 6). A decrease in endpoint E-selectin concentrations was observed after the consumption of the FXCO diet compared with the WD control $(P=0.023)$, however, not in comparison with the HOCO diet $(P=0.34)$. No significant changes were observed in endpoint concentrations for SVCAM-1 and sICAM-1 between the treatment groups (Table 6). As compared with the WD control, after the subjects consumed the FXCO diet, the change in endpoint E-selectin concentrations was directly associated with changes in TC $(r$ 0.413; $P=0.012)$, LDL-cholesterol $(r 0.383 ; P=0.021)$ and non-HDL-cholesterol ( $r \quad 0.340 ; \quad P=0.042)$ concentrations (Table 7). However, changes in E-selectin concentrations following the consumption of the FXCO diet compared with the WD control did not correlate with other lipid parameters or plasma fatty acid concentrations (data not shown). There were no correlations between changes in lipid concentrations after the consumption of the HOCO diet and changes in inflammatory biomarkers.

\section{Intima-media thickness assessment}

A subset of sixteen subjects (age, 48.7 (s巨m 11.9) years; BMI, 30.53 (SEm 4.64$) \mathrm{kg} / \mathrm{m}^{2}$; four males and twelve females (four premenopausal)) completed the assessment of common carotid IMT. Two subjects withdrew due to relocation of residence. For endpoint measures, there were no significant changes detected in right posterior wall or left posterior wall average or maximum IMT between the dietary treatments or from baseline values at the study entry (Table 6).

Table 7. Correlation coefficients among the change in plasma E-selectin and the changes in serum lipids when the subjects consumed the flaxseed and high-oleic rapeseed oil diet compared with the Western diet*

\begin{tabular}{lcc}
\hline & \multicolumn{2}{c}{$\Delta$ E-selectin } \\
\cline { 2 - 3 } & $r$ & $P$ \\
\hline$\Delta$ Total cholesterol $(\mathrm{mmol} / \mathrm{l})$ & 0.413 & 0.012 \\
$\Delta$ LDL-cholesterol $(\mathrm{mmol} / \mathrm{l})$ & 0.383 & 0.021 \\
$\Delta$ HDL-cholesterol $(\mathrm{mmol} / \mathrm{l})$ & 0.218 & 0.202 \\
$\Delta \mathrm{TAG}(\mathrm{mmol} / \mathrm{l})$ & 0.055 & 0.751 \\
$\Delta$ Total:HDL-cholesterol & 0.211 & 0.216 \\
$\Delta$ LDL:HDL-cholesterol & 0.246 & 0.148 \\
$\Delta$ Non-HDL-cholesterol & 0.340 & 0.042 \\
\hline * Pearson correlation analyses were conducted to test associations.
\end{tabular}




\section{Discussion}

The present results are the first to demonstrate the lipidlowering efficacy of low-SFA diets enriched with novel HOCO alone or blended with ALA-rich flaxseed oil. Compared with the WD control, we observed substantial decreases in TC for both the HOCO and the FXCO diets after $28 \mathrm{~d}$, with the FXCO diet further reducing TC beyond that of the HOCO diet (Table 5; Fig. 1). The present study observed similar reductions in LDL-cholesterol concentrations after the consumption of the HOCO and FXCO diets compared with the WD control. Reports examining the lipid-lowering action of PUFA-rich $v$. MUFA-rich diets support the notion that PUFA-rich diets reduce TC and LDL-cholesterol concentrations comparable with MUFA-rich diets, and that PUFA oils elicit a slight TAGlowering effect ${ }^{(2,20-22)}$. Similarly, compared with the HOCO diet, the FXCO diet and WD control, both higher in dietary PUFA content, tended to reduce endpoint TAG concentrations; however, due to large individual variation, there was no difference in percentage change in TAG levels from baseline between the dietary interventions examined.

The ability of HOCO to reduce TC and LDL-cholesterol, as well as to preserve HDL-cholesterol, is of particular interest since to date the efficacy of HOCO in modulating blood lipids has not been assessed. Furthermore, it has previously been reported that not all MUFA-rich oils elicit the same effects on plasma cholesterol concentrations ${ }^{(23)}$, suggesting the importance of other oil-derived fatty acid and non-lipid components. Reports suggest that ALA-rich flaxseed oil interventions fail to modify TC and LDL-cholesterol levels when compared with other dietary interventions ${ }^{(14,15)}$. However, these results could be confounded by the use of MUFA and $n$-6 PUFA dietary controls. Limited work has directly compared dietary flaxseed oil with MUFA-rich oils. Whereas Singer et al. ${ }^{(24)}$ observed a reduction in TAG, as well as in TC and LDL-cholesterol levels after 2-week supplementation with $60 \mathrm{ml} / \mathrm{d}$ of flaxseed oil but not with olive oil, Li et al. ${ }^{(25)}$ failed to find differences in plasma lipids after 4-week supplementation of a rapeseed oil or flaxseed oil-enriched diet. In the present study, substitution of $50 \%$ HOCO with flaxseed oil in the FXCO treatment group was effective in further reducing TC compared with the HOCO treatment group.

FXCO reduced HDL-cholesterol from baseline, resulting in lower endpoint HDL-cholesterol levels than the WD control (Table 5; Fig. 1). Previous studies administering high doses of flaxseed oil to hypercholesterolaemic subjects have observed reductions in HDL-cholesterol levels ${ }^{(26-29)}$. Generally, dietary strategies replacing SFA with PUFA result in a reduction in plasma TC and LDL-cholesterol and a parallel decrease in plasma HDL-cholesterol concentrations. Although concern exists that the cardioprotection associated with low LDL-cholesterol is diminished with simultaneous reductions in HDL-cholesterol, it has been shown that rates of cholesterol efflux from macrophage cells to serum are not affected $^{(30)}$. Furthermore, endpoint LDL:HDL-cholesterol ratios were reduced in response to the HOCO and FXCO diets compared with the WD control (Table 5). The LDL:HDL-cholesterol ratio is valuable in evaluating CVD risk across many populations ${ }^{(31)}$. As well, non-HDL-cholesterol provides a single measure of the atherogenic apo Bcontaining lipoproteins and can thus provide a tool for cardiovascular risk assessment ${ }^{(6,31)}$. After the consumption of the FXCO diet, non-HDL-cholesterol levels decreased beyond that of the HOCO diet and the WD control. Therefore, the additive effects of ALA and oleic acid in the FXCO diet may have provided additional hypolipidaemic effects that extend beyond those incurred by the HOCO diet alone.

In addition to dyslipidaemia, elevated CRP levels associate with clinical manifestations of atherosclerosis and CVD risk $^{(10)}$. The intricate communication between inflammatory stimuli and endothelial cell adhesion molecules regulates inflammatory responses and the progression of atherosclerosis $^{(13)}$. Thus, a direct association may exist between plasma concentrations of VCAM-1, ICAM-1 and E-selectin and the extent of atherosclerosis and incidence of CVD risk ${ }^{(11,12)}$. In vitro studies have shown the ability of oleic acid to inhibit cytokine-induced expression of VCAM-1, ICAM-1 and E-selectin in endothelial cells ${ }^{(32,33)}$. Although human clinical trials have yet to specifically investigate effects of HOCO on inflammatory biomarkers, Keogh et $a l^{\left({ }^{(3)}\right.}$ failed to observe any effect of a MUFArich diet on serum CRP or plasma adhesion molecules in forty healthy adults. Likewise, the consumption of the HOCO-rich diet for $28 \mathrm{~d}$ did not affect inflammatory biomarker measures. Results of the clinical trials investigating the effects of flaxseed oil on inflammatory biomarkers and adhesion molecules are inconsistent ${ }^{(14)}$. It has been suggested that the discrepancy may be dose related, as intakes exceeding $14 \mathrm{~g} / \mathrm{d}$ of ALA from flaxseed oil have been shown to be more effective. In the present study, after 4-week supplementation of $21 \mathrm{~g} / \mathrm{d}$ ( $7.5 \%$ energy) of ALA in the FXCO diet, a reduction was observed in E-selectin compared with the WD control; however, no reductions in other inflammatory biomarkers were observed. In a 6-week randomised crossover trial that examined hypercholesterolaemic subjects consuming 6.5\% ALA supplemented from walnuts and flaxseed oil daily, Zhao et al. ${ }^{(28)}$ observed significant reductions in serum CRP, VCAM-1, ICAM-1 and E-selectin, compared with an average American diet. Similarly, decreases in CRP, VCAM-1, as well as in IL-6, have been reported with the supplementation of $15 \mathrm{ml} / \mathrm{d}$ flaxseed oil $(8 \cdot 1 \mathrm{~g} \mathrm{ALA} / \mathrm{d})$ for 12 weeks $^{(26,35,36)}$; however, no effects on ICAM-1 or E-selectin were observed ${ }^{(35,36)}$. In contrast, recently Nelson et $a l .{ }^{(37)}$ failed to observe decreases in CRP or IL-6 in healthy abdominally obese subjects consuming $5 \%$ of energy from ALA for 8 weeks. Similar to the latter study, we observed no change in plasma CRP or IL-6 concentration following the consumption of the FXCO diet. 
Unlike VCAM-1 and ICAM-1, E-selectin activity is specific to the surface of stimulated endothelial cells, mediating the rolling of monocytes along the cell surface ${ }^{(38)}$. Furthermore, the expression of E-selectin directly associates with dyslipidaemia. It was previously shown that effective lipid-lowering intervention reduced plasma E-selectin concentrations in dyslipidaemic subjects; however, the lipid-lowering effect was not associated with a reduction in VCAM-1 or ICAM- ${ }^{(39)}$. Of interest, in the present study, a significant correlation was observed between changes in plasma E-selectin and TC, LDL-cholesterol and non-HDL-cholesterol concentrations when subjects consumed the FXCO diet compared with the WD control (Table 7). However, albeit the reduction in serum lipids following the consumption of the FXCO and HOCO diets, there was no change in VCAM-1 or ICAM-1 concentrations. Since the FXCO diet resulted in reductions in TC and nonHDL-cholesterol concentrations beyond that of the HOCO diet (Table 5), we speculate that the acute effects of FXCO consumption on E-selectin concentrations may be attributed to the magnitude of reductions in circulating lipids.

The discrepancy between the present results and those of previous studies that reported reductions in inflammatory biomarkers may be related to subject baseline levels of those biomarkers. In the present study, the subject baseline levels of inflammatory biomarkers CRP and IL-6 were in the healthy range compared with those of subjects examined previously ${ }^{(26,35,40)}$. Similarly, studies that failed to observe an effect of ALA intervention on inflammatory biomarkers have attributed the absence of response to a 'floor effect'; the inability to detect changes due to low baseline levels ${ }^{(37,41)}$. Another consideration may be the duration of the present study. Although a 4 -week intervention is typically sufficient to observe significant alterations in blood lipids, previous studies reporting reductions in inflammatory markers were of 6-12 weeks in duration $^{(26,28,35,36)}$. Similarly, the limited study duration may also explain the absence of treatment effects on carotid IMT. Bemelmans et al. $^{(40)}$, using a parallel-arm design and a 2-year dietary intervention, found that $4.5 \mathrm{~g} / \mathrm{d}$ of ALA yielded no significant effect on IMT progression. The present study focused on examining whether a high dose of ALA, approximately 3.5-fold greater than that used in Bemelmans et $a l^{(40)}$, utilising a crossover design, would have acute effects on IMT progression; however, no positive action was observed.

The plasma fatty acid concentrations reflected fatty acid profiles of the experimental oils, indicating compliance with the dietary interventions ${ }^{(42,43)}$. After consumption of the ALA-rich FXCO diet, an approximate 5-fold increase in plasma ALA (18:3n-3) concentrations and 3-fold increase in EPA $(20: 5 n-3)$ concentrations were observed compared with the HOCO diet and WD control. However, there were no differences in plasma DHA (20:6n-3) concentrations between the FXCO diet and the WD control. These results are consistent with previous stable isotope tracer studies demonstrating the linear relationship between dietary ALA intakes and plasma EPA, with no direct relationship between ALA intakes and plasma DHA due to limited conversion rates ${ }^{(44)}$. Nonetheless, the increase in plasma concentration of ALA, EPA and DPA after the consumption of the FXCO diet may be cardioprotective as an inverse association has been found between plasma concentrations of combined EPA and DHA, as well as of ALA, and risk of fatal IHD ${ }^{(45)}$. Furthermore, the higher plasma MUFA concentration after the consumption of the HOCO diet may provide cardiovascular benefits, as MUFA has been shown to be resistant to oxidative modifications of LDL-cholesterol ${ }^{(46)}$.

A potential limitation of the present study is that the experimental diets were not balanced for dietary cholesterol levels; however, it has been reported that in human subjects, dietary fatty acids are primary determinants of serum cholesterol, whereas dietary cholesterol has minimal effect on modulating serum cholesterol levels ${ }^{(3,47,48)}$. Furthermore, the average daily intake of cholesterol in each experimental diet was considerably lower than the AHA recommendation of $<300 \mathrm{mg} / \mathrm{d}^{(9)}$. Moreover, the feasibility of incorporating both HOCO and FXCO into typical diets requires further consideration. In order to maintain total fat energy intake, it is crucial to target fat substitution $v$. fat supplementation of the diet. The high-stability properties of HOCO make it a practical substitution for trans fat-rich partially hydrogenated vegetable oils in food processing, frying and culinary purposes $^{(8)}$. Increased dietary ALA intake can be achieved by fortifying dressings, spreads and margarines with the FXCO blend as a replacement of traditional products. Currently, the US Food and Drug Administration (FDA) has authorised a qualified health claim stating that rapeseed (canola) oil (approximately $19 \mathrm{~g} / \mathrm{d}$ ) may reduce the risk of CHD due to its unsaturated fat content, recommending direct energetic replacement of dietary SFA with rapeseed (canola) oil ${ }^{(49)}$. Therefore, increased compliance with dietary recommendations and targeting a reduction in CHD risk would be possible by replacing a proportion of commonly used dietary oils and spreads in the WD with HOCO alone or blended with flaxseed oil.

In conclusion, the present study is the first human clinical trial to investigate effects of HOCO on serum lipids and other markers of CVD risk. HOCO alone or when blended with flaxseed oil effectively reduced serum TC and LDLcholesterol compared with a WD. Moreover, the ALA-rich FXCO may further target inflammation and atherogenic pathways by reducing plasma E-selectin. Substitution of dietary fats common to the WD with both HOCO and flaxseed oil is a feasible option to target dietary recommendations and risk factors for CVD.

\section{Acknowledgements}

We thank the personnel at the Richardson Centre for Functional Foods and Nutraceuticals including Kimberley 
Robinson as a clinical coordinator, Darren Speziale, Katherine Leung and the other staff of the Metabolic Kitchen for preparation of the controlled meals, and Joan Richardson and ultrasound sonographers at St Boniface General Hospital for conducting the carotid ultrasound scans. The present work was funded in part by Flax Canada 2015, Canola Council of Canada and Agri-Food Research \& Development Initiative. We acknowledge the kind contribution of the flaxseed oil and the HOCO from Bioriginal Food \& Science Corporation and Dow AgroSciences LLC, respectively. L. G. G. was supported by a doctoral studentship award from the Manitoba Health Research Council. P. J. H. J. was responsible for the conception and design of the project, submission for ethical approval and seeking financial support. L. G. G. was responsible for subject recruitment, management of the clinical trial, data collection and laboratory analysis, statistical analysis and writing the manuscript. J. A. G. contributed to subject recruitment and was clinical coordinator for the trial. S.-Y. H. and D. S. J. coordinated and analysed IMT scans. All the authors contributed to revisions of the manuscript and reviewed the final version. The authors declare no conflict of interest.

\section{References}

1. Mente A, de Koning L, Shannon HS, et al. (2009) A systematic review of the evidence supporting a causal link between dietary factors and coronary heart disease. Arch Intern Med 169, 659-669.

2. Mensink RP, Zock PL, Kester AD, et al. (2003) Effects of dietary fatty acids and carbohydrates on the ratio of serum total to HDL cholesterol and on serum lipids and apolipoproteins: a meta-analysis of 60 controlled trials. Am J Clin Nutr 77, 1146-1155.

3. Hu FB, Manson JE \& Willett WC (2001) Types of dietary fat and risk of coronary heart disease: a critical review. $J \mathrm{Am}$ Coll Nutr 20, 5-19.

4. Eckel RH, Kris-Etherton P, Lichtenstein AH, et al. (2009) Americans' awareness, knowledge, and behaviors regarding fats: 2006-2007. J Am Diet Assoc 109, 288-296.

5. Hu FB, Stampfer MJ, Manson JE, et al. (1999) Dietary intake of alpha-linolenic acid and risk of fatal ischemic heart disease among women. Am J Clin Nutr 69, 890-897.

6. National Cholesterol Education Program (NCEP) Expert Panel on Detection, Evaluation, and Treatment of High Blood Cholesterol in Adults (Adult Treatment Panel III) (2002) Third Report of the National Cholesterol Education Program (NCEP) Expert Panel on Detection, Evaluation, and Treatment of High Blood Cholesterol in Adults (Adult Treatment Panel III) final report. Circulation 106, 3143-3421.

7. Kris-Etherton PM, Taylor DS, Yu-Poth S, et al. (2000) Polyunsaturated fatty acids in the food chain in the United States. Am J Clin Nutr 71, 179S-188S.

8. Tarrago-Trani MT, Phillips KM, Lemar LE, et al. (2006) New and existing oils and fats used in products with reduced trans-fatty acid content. J Am Diet Assoc 106, 867-880.

9. American Heart Association Nutrition CommitteeLichtenstein AH, Appel LJ, et al. (2006) Diet and lifestyle recommendations revision 2006: a scientific statement from the American Heart Association Nutrition Committee. Circulation 114, $82-96$.
10. Ridker PM \& Silvertown JD (2008) Inflammation, C-reactive protein, and atherothrombosis. J Periodontol 79, 1544-1551.

11. Hwang SJ, Ballantyne CM, Sharrett AR, et al. (1997) Circulating adhesion molecules VCAM-1, ICAM-1, and E-selectin in carotid atherosclerosis and incident coronary heart disease cases: the Atherosclerosis Risk In Communities (ARIC) study. Circulation 96, 4219-4225

12. Blankenberg S, Rupprecht HJ, Bickel C, et al. (2001) Circulating cell adhesion molecules and death in patients with coronary artery disease. Circulation 104, 1336-1342.

13. Ross R (1999) Atherosclerosis - an inflammatory disease. $N$ Engl J Med 340, 115-126.

14. Prasad K (2009) Flaxseed and cardiovascular health. J Cardiovasc Pharmacol 54, 369-377.

15. Pan A, Yu D, Demark-Wahnefried W, et al. (2009) Metaanalysis of the effects of flaxseed interventions on blood lipids. Am J Clin Nutr 90, 288-297.

16. Mifflin MD, St Jeor ST, Hill LA, et al. (1990) A new predictive equation for resting energy expenditure in healthy individuals. Am J Clin Nutr 51, 241-247.

17. Friedewald WT, Levy RI \& Fredrickson DS (1972) Estimation of the concentration of low-density lipoprotein cholesterol in plasma, without use of the preparative ultracentrifuge. Clin Chem 18, 499-502.

18. Folch J, Lees M \& Slone Stanley GH (1957) A simple method for the isolation and purification of total lipides from animal tissues. J Biol Chem 226, 497-509.

19. Lorenz MW, Markus HS, Bots ML, et al. (2007) Prediction of clinical cardiovascular events with carotid intima-media thickness: a systematic review and meta-analysis. Circulation 115, 459-467.

20. Kris-Etherton PM (1999) AHA science advisory: monounsaturated fatty acids and risk of cardiovascular disease. $J$ Nutr 129, 2280-2284.

21. Gardner CD \& Kraemer HC (1995) Monounsaturated versus polyunsaturated dietary fat and serum lipids. A meta-analysis. Arterioscler Thromb Vasc Biol 15, 1917-1927.

22. Dai J, Su YX, Bartell S, et al. (2009) Beneficial effects of designed dietary fatty acid compositions on lipids in triacylglycerol-rich lipoproteins among Chinese patients with type 2 diabetes mellitus. Metabolism 58, 510-518.

23. Truswell AS \& Choudhury N (1998) Monounsaturated oils do not all have the same effect on plasma cholesterol. Eur J Clin Nutr 52, 312-315.

24. Singer P, Jaeger W, Berger I, et al. (1990) Effects of dietary oleic, linoleic and alpha-linolenic acids on blood pressure, serum lipids, lipoproteins and the formation of eicosanoid precursors in patients with mild essential hypertension. $J$ Hum Hypertens 4, 227-233.

25. Li D, Sinclair A, Wilson A, et al. (1999) Effect of dietary alpha-linolenic acid on thrombotic risk factors in vegetarian men. Am J Clin Nutr 69, 872-882.

26. Rallidis LS, Paschos G, Liakos GK, et al. (2003) Dietary alphalinolenic acid decreases $\mathrm{C}$-reactive protein, serum amyloid A and interleukin-6 in dyslipidaemic patients. Atherosclerosis 167, 237-242.

27. Paschos GK, Zampelas A, Panagiotakos DB, et al. (2007) Effects of flaxseed oil supplementation on plasma adiponectin levels in dyslipidemic men. Eur J Nutr 46, 315-320.

28. Zhao G, Etherton TD, Martin KR, et al. (2004) Dietary alphalinolenic acid reduces inflammatory and lipid cardiovascular risk factors in hypercholesterolemic men and women. $J$ Nutr 134, 2991-2997.

29. Wilkinson P, Leach C, Ah-Sing EE, et al. (2005) Influence of alpha-linolenic acid and fish-oil on markers of cardiovascular risk in subjects with an atherogenic lipoprotein phenotype. Atherosclerosis 181, 115-124. 
30. Kralova Lesna I, Suchanek P, Kovar J, et al. (2008) Replacement of dietary saturated FAs by PUFAs in diet and reverse cholesterol transport. J Lipid Res 49, 2414-2418.

31. Fernandez ML \& Webb D (2008) The LDL to HDL cholesterol ratio as a valuable tool to evaluate coronary heart disease risk. J Am Coll Nutr 27, 1-5.

32. Carluccio MA, Massaro M, Bonfrate C, et al. (1999) Oleic acid inhibits endothelial activation: a direct vascular antiatherogenic mechanism of a nutritional component in the mediterranean diet. Arterioscler Thromb Vasc Biol 19, $220-228$.

33. Massaro M, Carluccio MA, Paolicchi A, et al. (2002) Mechanisms for reduction of endothelial activation by oleate: inhibition of nuclear factor-kappaB through antioxidant effects. Prostaglandins Leukot Essent Fatty Acids 67, $175-181$.

34. Keogh JB, Grieger JA, Noakes M, et al. (2005) Flow-mediated dilatation is impaired by a high-saturated fat diet but not by a high-carbohydrate diet. Arterioscler Thromb Vasc Biol 25, 1274-1279.

35. Paschos GK, Rallidis LS, Liakos GK, et al. (2004) Background diet influences the anti-inflammatory effect of alphalinolenic acid in dyslipidaemic subjects. Br J Nutr 92, 649-655.

36. Rallidis LS, Paschos G, Papaioannou ML, et al. (2004) The effect of diet enriched with alpha-linolenic acid on soluble cellular adhesion molecules in dyslipidaemic patients. Atherosclerosis 174, 127-132.

37. Nelson TL, Stevens JR \& Hickey MS (2007) Inflammatory markers are not altered by an eight week dietary alphalinolenic acid intervention in healthy abdominally obese adult males and females. Cytokine 38, 101-106.

38. Roldan V, Marin F, Lip GY, et al. (2003) Soluble E-selectin in cardiovascular disease and its risk factors. A review of the literature. Thromb Haemost 90, 1007-1020.

39. Hackman A, Abe Y, Insull W Jr, et al. (1996) Levels of soluble cell adhesion molecules in patients with dyslipidemia. Circulation 93, 1334-1338.
40. Bemelmans WJ, Lefrandt JD, Feskens EJ, et al. (2004) Increased alpha-linolenic acid intake lowers C-reactive protein, but has no effect on markers of atherosclerosis. Eur J Clin Nutr 58, 1083-1089.

41. Barcelo-Coblijn G, Murphy EJ, Othman R, et al. (2008) Flaxseed oil and fish-oil capsule consumption alters human red blood cell $n-3$ fatty acid composition: a multiple-dosing trial comparing 2 sources of $n-3$ fatty acid. Am J Clin Nutr 88, 801-809.

42. Baylin A \& Campos H (2006) The use of fatty acid biomarkers to reflect dietary intake. Curr Opin Lipidol 17, 22-27.

43. Hodson L, Skeaff CM \& Fielding BA (2008) Fatty acid composition of adipose tissue and blood in humans and its use as a biomarker of dietary intake. Prog Lipid Res 47, 348-380.

44. Burdge GC (2006) Metabolism of alpha-linolenic acid in humans. Prostaglandins Leukot Essent Fatty Acids 75 , 161-168.

45. Lemaitre RN, King IB, Mozaffarian D, et al. (2003) n-3 Polyunsaturated fatty acids, fatal ischemic heart disease, and nonfatal myocardial infarction in older adults: the Cardiovascular Health Study. Am J Clin Nutr 77, 319-325.

46. Moreno JA, Lopez-Miranda J, Perez-Martinez P, et al. (2008) A monounsaturated fatty acid-rich diet reduces macrophage uptake of plasma oxidised low-density lipoprotein in healthy young men. Br J Nutr 100, 569-575.

47. Grundy SM \& Denke MA (1990) Dietary influences on serum lipids and lipoproteins. J Lipid Res 31, 1149-1172.

48. Jones PJ, Pappu AS, Hatcher L, et al. (1996) Dietary cholesterol feeding suppresses human cholesterol synthesis measured by deuterium incorporation and urinary mevalonic acid levels. Arterioscler Thromb Vasc Biol 16, 1222-1228.

49. FDA U.S. Food and Drug Administration (2006) Qualified Health Claims: Letter of Enforcement Discretion - Unsaturated Fatty Acids from Canola Oil and Reduced Risk of Coronary Heart Disease (Docket no. 2006Q-0091). http://www.fda. gov/Food/LabelingNutrition/LabelClaims/QualifiedHealth Claims/ucm072958.htm (accessed August 2009). 